\title{
Electro-osmosis Route of Administration
}

National Cancer Institute

\section{Source}

National Cancer Institute. Electro-osmosis Route of Administration. NCI Thesaurus.

Code C38633.

Administration of drug by way of diffusion, through a membrane in an electric field.

Electro-osmosis describes the active transport of non-ionized solutes associated with the bulk flow of fluid in the direction of the counter-ions that occurs with iontophoretic transport of other, ionized solutes. Using this method, even a neutral compound can be administered. Method can be used for local and systemic drug delivery. 